\title{
Effects of Simulation-based SBAR Education Program on Communication Clarity and Communication Self-Confidence in Nursing Students
}

\author{
Hye Kyung $\mathrm{Oh}^{1}$ \\ ${ }^{1}$ Associate Professor, Dept. of Nursing, Daegu University, South Korea, kyungoh@daegu.ac.kr
}

\begin{abstract}
This study verified the effects of the Situation, Background, Assessment, Recommendation (SBAR) education program using simulation on nursing students' communication clarity and communication self-confidence. A one group pre- and post-test quasi-experimental research design was used. The participants were comprised of 82 fourth-year nursing students (17 men and 65 women) enrolled in a simulation course. Data were analyzed using SAS 9.2. Pre-post differences in communication clarity and communication self-confidence before and after the simulation-based communication education were analyzed by conducting a t-test. Communication clarity and communication self-confidence according to participants' general characteristics were analyzed by conducting a t-test and ANOVA, followed by a Scheffe post-hoc test. The results revealed that there were statistically significant differences in communication clarity and communication self-confidence between pre- and post-SBAR education program using emergency patient care simulation. This study obtained the following findings: The mean score for communication clarity statistically significantly improved after the simulation-based SBAR education program from 3.84 to 4.17 ( $\mathrm{t}=5.86, \mathrm{p}<.0001)$. In this study, communication self-confidence statistically significantly improved after the SBAR education program from 6.49 points to $7.18(\mathrm{t}=5.53, \mathrm{p}=<.0001)$. Nursing students improved their communication clarity and communication self-confidence suitable for complex and emergency clinical situations through the SBAR education program using simulation. Consequently, the participants found it possible to communicate clearly and confidently as a clinical nurse after graduation, collaborate with medical teams, and improve patient safety and nursing quality.
\end{abstract}

Keywords: SBAR, Simulation, Communication Clarity, Communication Self-Confidence

\section{Introduction}

\subsection{Necessity and Purpose}

Nurses' efficient communication in clinical practice is an important determinant of nursing quality[1]. In focus group interviews with 11 head nurses working at a large hospital, the additional job competency required for both novice nurses and training nurses was identified to be communication ability. In focus group interviews with 11 novice nurses, it was found that they had not been taught on the ways to communicate with medical staff doing a variety of jobs[2].

In the nursing curriculum, communication education is provided mainly for communication with patients with respect to treatment[3]. In the communication with healthcare professionals, nursing students find it difficult to deliver information accurately due to an inability to organize essential data 
regarding patient status[4].

Situation, Background, Assessment, Recommendation (SBAR) is one of the techniques used to increase communication clarity in clinical practice. The SBAR technique is an effective and standardized communication method developed to adequately communicate patent information for patient problem solving[3]. It has been reported that the use of SBAR in communication among nurses improved communication clarity and reduced the rate of accident reports due to missing information[5]. According to studies on the effect of communication based on SBAR, confidence was enhanced since it was easy to organize information and remember the data[6], and efficiency in performing nursing tasks was improved[7].

As SBAR has been proven to have such positive effects, the technique should be taught, not only to clinical nurses, but also to graduate nurses, who are nurses in training. Education and training on communication among healthcare professionals should start from nursing colleges where nurses in training are taught.

Recently, studies on nursing students in Korea have reported that communication clarity, confidence and self-efficacy[8][9], critical thinking[10][11], and clinical decision-making ability[12] were improved.

The previous studies conducted in Korea analyzed the effect on communication mainly using methods such as lecture, role play, and report writing. Studies that investigate the effect of SBAR using simulation in which diverse clinical cases can be experienced have been presented only recently.

The SBAR communication technique is taught more effectively by utilizing simulation[10]. Particularly, nursing students in Korea have very few opportunities to have direct experience of emergency situations in clinical practice. When nursing students lacking experience with emergency patients graduate and become clinical nurses, despite having adequate patient information, they may not effectively respond to the situation because they are unable to clearly communicate and deliver the patient information to other healthcare professionals or colleagues. Such ineffective communication is directly related to the risks of safety incidents that can occur at any time in clinical settings.

There are various methods to educate standardized SBAR communication. In this study, the researcher aimed to provide nursing students with standardized SBAR education based on simulation to allow them to obtain direct experience in simulated situations and adapt to urgent and complex patient situations. It is difficult to have direct experience of pre- and post-surgery emergency situations, and studies investigating the effects of the SBAR communication technique using simulations of actual cases of emergency patients, are still at an early stage. Nursing students will be able to learn effective communication techniques and efficient responses to emergency situations by directly and repeatedly practicing SBAR communication techniques with physicians and nursing colleagues in simulations with urgent and complex patients. With the experiences through the simulation, it is expected that nursing students will be able to communicate patient status clearly and confidently, and accurately determine and respond to the emergency situations.

This study examined the effects of the SBAR education program based on the simulation of pre- and post-surgery emergency situations on communication clarity and communication self-confidence in graduate nurses.

\subsection{Hypothesis}

The hypotheses of this study examined the effects of the SBAR education program based on the simulation of emergency situations on communication clarity and communication self-confidence in graduate nurses. The hypotheses were as follows: 


\subsubsection{First Hypothesis}

Nursing students will improve their communication clarity after the SBAR education program based on the simulation of emergency situations.

\subsubsection{Second Hypothesis}

Nursing students will improve their communication self-confidence after the SBAR education program based on the simulation of emergency situations.

\section{Theoretical Background}

Nurses respond to various clinical situations through communication with many healthcare professionals in hospitals. Therefore, they must possess effective communication skills, and this can significantly contribute towards reducing the risks of safety incidents in clinical settings[13].

SBAR is a structured communication method, which includes the components of situation, background, assessment, and recommendation in relation to important health problems.

The structural components of SBAR enhance critical thinking in emergency situations and demonstrate the need to identify issues in the emergency situation and determine the solutions[14]. "Situation" is the stage when a speaker communicates what happened and why they need assistance from healthcare professionals, as well as the employee's affiliation, name, patient name, and patient's major symptoms. "Background" includes the patient's clinical background, reason for admission, medical status, and comorbidities, which enable a listener to understand the comprehensive picture of the patient's situation described in "situation." "Assessment" includes data related to the patient's presenting issue such as vital signs, quantitative data such as test results and qualitative data and requires a clinical judgement of the patient's status. "Recommendation" includes suggesting a specific request and obtaining the listener's opinions to receive orders for necessary interventions[15].

Kesten (2011) [3] reported that nursing students' ability to use the SBAR technique increased after receiving the SBAR lecture and performing a roleplay. Yu and Kang's (2017)[16] study showed that although the level of satisfaction of education in nursing students increased after the role play, it did not impact their communication clarity and confidence.

In South Korea, education through SBAR lectures, video, and role play were reported to be effective in enhancing communication clarity, critical thinking, and confidence[8][17][18]. Recent studies reported that communication clarity and confidence improved when individuals utilized and repeatedly practiced SBAR communication techniques in simulations involving direct experience of patient situations[10][12].

Accordingly, of the various SBAR education methods, this study selected the simulation method to allow nursing students, who have limited experience in handling complex patient situations, to experience and learn through realistic simulations of urgent and complex situations. Therefore, the researcher aimed to verify the effect of enhanced communication clarity and confidence through direct experience and repeated learning from the simulations.

\section{Methods}

\subsection{Design}

This is a quasi-experimental study with one group pretest-posttest design, conducted to investigate the effects of simulation-based SBAR communication education on communication clarity and communication self-confidence of nursing students. The present study was performed with a single 
group, because simulation-based SBAR communication education was provided to the students enrolled in a simulation course and therefore, it was not possible to create a separate control group.

\subsection{Participants}

Study participants were fourth-year nursing students taking a simulation course. As this study simulated complex emergency patient situations, fourth-year nursing students with general expert knowledge of pre- and post-surgery emergency patient situations and direct clinical experiences were selected. Prior to the study, the students were informed of the study purpose and procedure, anonymity, and confidentiality. After verbally explaining that the class would not be affected by participation in the survey and survey results and that there would be no disadvantage if they refused to participate or withdrew, the students who voluntarily consented to participate signed a written consent form. A total of 82 students who voluntarily consented to participate in the study were selected. The sample size was chosen using $\mathrm{G}^{*}$ power 3.1 based on the assumptions of a significance level $(\alpha)$ for two-tailed testing of.05, a power (1- $\beta$ ) of.8, and an effect size (d) of.315.

\subsection{SBAR Education Program}

Simulation-based communication education was provided over 30 hours for a total of eight weeks, operated for 4 hours every week for 7 weeks. The last week was operated for 2 hours for evaluation. The simulated clinical situations depicted patient cases before and after surgery and each of the cases was either a simple situation in which the patient complained of pain or a complex situation in which the patient complained of complicated problems such as water-electrolyte imbalance and transfusion reaction. Patient cases were chosen in consideration of the level of theoretical education received by the study participants. The contents of the simulation-based communication education are presented in [Table 1].

For the simulation-based communication education, participants were randomly assigned to a team of 4-5 members. They listened to lectures regarding ways to apply SBAR, the nursing skills required for each patient case/illness, and were given the opportunity to practice the nursing skills. In weekly sessions, each team performed a problem analysis for the nursing cases and discussed nursing activities to resolve the problems, the prioritization of nursing activities, role division among team members, and the design of SBAR communication. Nursing care and SBAR communication were performed in simulation situations in which emergency patient cases were reproduced. Subsequently, the teams were engaged in reflective learning while watching a video of their performances. After team activities were completed, the entire class participated in a session for reflective learning.

[Table 1] SBAR Education Program Using Simulation

\begin{tabular}{|c|c|c|}
\hline Week & \multicolumn{2}{|c|}{ Content } \\
\hline 1 & \multicolumn{2}{|c|}{ Orientation and pre-test } \\
\hline $2 \sim 4$ & $\begin{array}{c}\text { SBAR Introduction and role play } \\
\text { Nursing skill practice }\end{array}$ \\
\hline $5 \sim 7$ & $\begin{array}{c}\text { Simulation and } \\
\text { debriefing }\end{array}$ & $\begin{array}{c}\text {-Nursing care and SBAR planning } \\
\text {-Nursing care and SBAR debriefing }\end{array}$ \\
\hline 8 & \multicolumn{2}{|c|}{ Post-test } \\
\hline
\end{tabular}




\subsection{Research Instruments}

\subsubsection{Communication Clarity}

To assess communication clarity and content in medical students, a Korean version of the 20-item tool developed by Marshall, Harrison, and Falnagan (2009)[19] was used. The Korean version was translated and modified to comprise a total of 14 items by Cho (2013)[17]. The items were rated on a 5point scale and the total scores ranged from 14 to 70 points. The higher the score, the higher the communication ability. In this study, the Cronbach's alpha for the reliability of the tool was $\alpha=.93$.

\subsubsection{Communication Confidence}

Communication confidence refers to the level of confidence with which to report a clinical situation to medical staff. In this study, communication confidence was assessed using an instrument developed by Kim (2018)[11]. The instrument consisted of five items on a 10-point scale, with the total scores ranging from 5 to 50 points. The higher the score, the higher the communication confidence. In this study, the Cronbach's alpha for the reliability of the tool was $\alpha=.96$.

\subsection{Data Collection and Analysis}

This study included a single group of participants who received the SBAR education program based on the simulation of emergency situations during a 15-week education curriculum, therefore, the results should be interpreted with caution. Data were collected between April and June 2020, and were analyzed using SAS 9.2. Participant characteristics, communication clarity, and communication self-confidence were examined by computing frequencies, percentages, means, and standard deviations. Pre-post differences in communication clarity and communication self-confidence before and after the simulation-based communication education were analyzed by conducting t-test. Communication clarity and communication self-confidence according to the participants' general characteristics were analyzed by conducting a t-test and ANOVA followed by the Scheffe post-hoc test.

\subsection{Ethical Considerations}

For ethical considerations on behalf of study participants, the study was approved by the bioethics committee of A University located in B Metropolitan City (1040621-202004-HR-005).

The study was conducted with fourth year nursing students taking a simulation course, who were informed of study purpose and procedure, anonymity, and confidentiality prior to participation. In addition, the students were fully informed that participation in the survey and survey results would not affect the class and that refusal to participate or withdrawal would not result in any disadvantage. Those who agreed to voluntarily participate signed a written consent form.

\section{Results}

\subsection{Characteristics of Participants}

General characteristics of the participants are reported in [Table 2]. A total of 39 (47.56\%) of the participants were aged 21 or younger, which was the largest age group. A total of 24 participants $(29.28 \%)$ were 23 or older, and 19 (23.17\%) were 22 years old. A total of 59 students $(71.95 \%)$ selfassessed that they had a positive personality, and $62(75.61 \%)$ responded that they were highly capable of adapting to situational and environmental changes. A total of 37 students $(54.88 \%)$ were satisfied with college life, $52(63.41 \%)$ were satisfied with nursing as the major area of study, and $45(54.88 \%)$ 
were satisfied with clinical practice.

[Table 2] Characteristics of Participants $(\mathrm{N}=82)$

\begin{tabular}{|c|c|c|}
\hline \multicolumn{2}{|c|}{ Categories } & $N(\%)$ \\
\hline \multirow{3}{*}{ Age(years) } & $\leq 21$ & $39(47.56)$ \\
\hline & 22 & $19(23.17)$ \\
\hline & $\geq 23$ & $24(29.28)$ \\
\hline \multirow{2}{*}{ Gender } & Female & $65(79.27)$ \\
\hline & Male & $17(20.73)$ \\
\hline \multirow{3}{*}{ Personality } & Positive & $59(71.95)$ \\
\hline & Ordinary & $20(24.39)$ \\
\hline & Negative & $3(3.66)$ \\
\hline \multirow{3}{*}{$\begin{array}{l}\text { Adaptation to the situation and } \\
\text { environmental Change }\end{array}$} & Very well & $62(75.61)$ \\
\hline & Moderate & $18(21.95)$ \\
\hline & Not & $2(2.44)$ \\
\hline \multirow{3}{*}{ Satisfaction of college life } & Satisfaction & $37(54.88)$ \\
\hline & Moderate & $45(45.12)$ \\
\hline & Not & - \\
\hline \multirow{3}{*}{ Satisfaction of nursing major } & Satisfaction & $52(63.41)$ \\
\hline & Moderate & $30(36.59)$ \\
\hline & Not & - \\
\hline \multirow{3}{*}{ Satisfaction of clinical practice } & Satisfaction & $45(54.88)$ \\
\hline & Moderate & $36(43.90)$ \\
\hline & Not & $1(1.22)$ \\
\hline
\end{tabular}

\subsection{Hypothesis Verification}

\subsubsection{First Hypothesis}

Nursing students will improve their communication clarity after the SBAR education program based on the simulation of emergency situations.

Matched sample t-test was conducted to test whether communication clarity changed between before and after the simulation-based SBAR education [Table 3]. Regarding communication clarity, posteducation score was higher, and the pre-post difference was statistically significant $(t=5.86, p=<.0001)$. The first hypothesis was supported by the improvement in communication clarity after learning than before the SBAR education program based on the simulation of emergency situations.

\subsubsection{Second Hypothesis}

Nursing students will improve their communication self-confidence after the SBAR education program based on the simulation of emergency situations.

Matched sample t-test was conducted to test whether communication self-confidence changed between before and after the simulation-based SBAR education [Table 3]. Regarding communication 
self-confidence, post-education score was also higher, and the pre-post difference was statistically significant $(\mathrm{t}=5.53, \mathrm{p}=<.0001)$. The second hypothesis was supported by the improvement in communication self-confidence after learning than before the SBAR education program based on the simulations of emergency situations.

[Table 3] Difference in Communication Clarity and Confidence between pre- and post-tests

\begin{tabular}{|c|c|c|c|}
\hline Variable & Pre-test & Post-test & \multirow{2}{*}{$\mathrm{t}(\mathrm{p})$} \\
\cline { 2 - 3 } & MSD & MSD & \multirow{2}{*}{$\begin{array}{c}5.86 \\
\left(<.0001^{* * *}\right)\end{array}$} \\
\hline Communication clarity & 3.840 .50 & 4.170 .51 & $\begin{array}{c}5.53 \\
\left(<.0001^{* * *}\right)\end{array}$ \\
\hline Communication confidence & 6.491 .56 & 7.181 .31 & \\
\hline$*<.05, * *<.01, * * *<.001$ & \multicolumn{2}{|c}{}
\end{tabular}

\subsection{Communication Clarity and Confidence According to Characteristics}

Communication clarity and communication self-confidence according to participants' general characteristics are reported in [Table 4]. Communication clarity did not show statistically significant differences according to any of the general characteristics. Communication self-confidence did not show statistically significant differences according to age and gender of the general characteristics. On the other hand, communication self-confidence showed statistically significant differences according to personality $(F=4.56, p=.0133)$, adaptability to situational and environmental changes $(F=6.56, p=.0023)$, satisfaction with nursing as the major area of study $(\mathrm{t}=3.15, \mathrm{p}=.0023)$, and satisfaction with clinical practice $(\mathrm{F}=5.34, \mathrm{p}=.0067)$. Scheffe post-hoc tests revealed that the score for communication selfconfidence was higher in students with positive personality than those with ordinary personality, in students with high level of adaptability to situational and environmental changes than those with moderate levels of adaptability, and in students highly satisfied with nursing as the major area of study compared to those who were moderately satisfied. In addition, the score was higher in students highly satisfied with clinical practice compared to those who were moderately satisfied.

[Table 4] Communication Clarity and Confidence according to Characteristics $(\mathrm{N}=82)$

\begin{tabular}{|c|c|c|c|c|c|}
\hline \multirow{2}{*}{\multicolumn{2}{|c|}{ Categories }} & \multicolumn{2}{|c|}{ Communication Clarity } & \multicolumn{2}{|c|}{ Communication Confidence } \\
\hline & & $\mathrm{M} \pm \mathrm{SD}$ & $F$ or $t(p)$ & $\mathrm{M} \pm \mathrm{SD}$ & $F$ or $t(p)$ \\
\hline \multirow{3}{*}{ Personality } & Positivea & $4.23 \pm 0.51$ & \multirow{3}{*}{$\begin{array}{c}1.76 \\
(.1794)\end{array}$} & $7.44 \pm 1.22$ & \multirow{3}{*}{$\begin{array}{c}4.56 \\
\left(.0133^{*}\right) \\
\mathrm{a}>\mathrm{b}\end{array}$} \\
\hline & Ordinaryb & $4.05 \pm 0.52$ & & $6.49 \pm 1.41$ & \\
\hline & Negativec & $3.79 \pm 0.33$ & & $6.67 \pm 0.31$ & \\
\hline \multirow{3}{*}{$\begin{array}{c}\text { Adaptation to } \\
\text { the Situation and } \\
\text { Environmental Change }\end{array}$} & Very wella & $4.24 \pm 0.49$ & \multirow{3}{*}{$\begin{array}{c}2.49 \\
(.0896)\end{array}$} & $7.46 \pm 1.23$ & \multirow{3}{*}{$\begin{array}{c}6.56 \\
(.0023 * *) \\
a>b\end{array}$} \\
\hline & Moderateb & $3.97 \pm 0.54$ & & $6.34 \pm 1.17$ & \\
\hline & Notc & $3.82 \pm 0.76$ & & $6.10 \pm 1.56$ & \\
\hline \multirow{2}{*}{ Satisfaction of nursing major } & Satisfactiona & $4.25 \pm 0.47$ & \multirow{2}{*}{$\begin{array}{c}1.88 \\
(.0642)\end{array}$} & $7.51 \pm 1.14$ & \multirow{2}{*}{$\begin{array}{c}3.15 \\
\left(.0023^{* *}\right)\end{array}$} \\
\hline & Moderateb & $4.03 \pm 0.57$ & & $6.61 \pm 1.40$ & \\
\hline \multirow{3}{*}{ Satisfaction of clinical practice } & Satisfactiona & $4.23 \pm 0.46$ & \multirow{3}{*}{$\begin{array}{c}0.99 \\
(.3767)\end{array}$} & $7.59 \pm 1.07$ & \multirow{3}{*}{$\begin{array}{c}5.34 \\
\left(.0067^{* *}\right) \\
\mathrm{a}>\mathrm{b}\end{array}$} \\
\hline & Moderateb & $4.11 \pm 0.57$ & & $4.69 \pm 1.43$ & \\
\hline & Notc & 3.71 & & 6.60 & \\
\hline
\end{tabular}

$*<.05, * *<.01$, post-analysis Scheffe test 


\section{Discussion}

This study examined the effects of the simulation-based SBAR education program on communication clarity and communication self-confidence of nursing students with following findings:

The mean score for communication clarity was statistically significantly improved after the simulation-based SBAR education program vs. before, from 3.84 to 4.17. This is consistent with the findings of previous studies in which simulation-based SBAR education programs were provided to graduate nurses[11][12]. Yoon and Lee (2018)[10] applied SBAR communication using team simulation to second-year nursing students. The study revealed that communication clarity significantly increased, from 3.32 points to 3.68 .

Communication clarity refers to accurate understanding by the listener of the information the speaker intends to deliver[20]. Enhanced communication clarity can prevent safety accidents by promoting appropriate nursing care. It can also positively influence job satisfaction and cooperation in clinical practice.

Inexperienced nurses experience stress and fear when verbally communicating with medical staff regarding patient status and may miss out useful patient information and make communication mistakes[13]. Nurses should be provided with communication education and training to strengthen communication clarity when they communicate with healthcare professionals, from the time when they are nursing students, that is, nurses in training. It is believed that by increasing clarity in communication with other types of healthcare professionals through an SBAR education program, novice nurses can overcome communication conflict that may occur while they acclimate themselves with clinical practice, as well as decrease and prevent patient safety accidents.

In this study, communication self-confidence was statistically significantly improved after the SBAR education program vs. before, from 6.49 points to 7.18 . This is in line with the findings of previous studies in which simulation-based SBAR education programs were provided [8][11][12].

Nurses interact with diverse types of healthcare professionals and play the role of a mediator between medical staff and patients. Accordingly, it is necessary for them to express their opinions clearly and confidently in communicating with others[13]. Kim (2018)[11] found that in simulations of emergency situations involving chest pain patients and respiratory failure patients, communication self-confidence was significantly improved in the experimental group who used the SBAR communication technique vs. the control group who used general communication handouts. In the current study, in emergency situations likely to occur before or after surgery, self-confidence in inter-medical staff communication was also enhanced. It is speculated that repeated experience with the SBAR communication technique helped study participants reduce anxiety and tension even in emergency situations reproduced in the simulation and accurately analyze the information to deliver, which led to an increase in communication self-confidence.

The analysis on communication self-confidence according to participants' general characteristics revealed statistically significant differences in personality, adaptability to situational and environmental changes, and satisfaction with nursing as the major area of study and with clinical practice. No other studies have used the same instrument used herein and thus, direct comparison cannot be made. However, the finding is similar to that of Lee (2015)[21], in which nursing students' communication ability was examined according to general characteristics. Lee (2015)[21] conducted a study with 320 third and fourth-year nursing students and found that communication ability was higher in students highly satisfied with college life, nursing as the major area of study, and clinical practice, than moderately satisfied students. It is believed that in nursing students (that is, nurses in training) who were highly satisfied with nursing as the major area of study and with clinical practice, their communication ability may also have been influenced by enthusiasm about job performance and achievement.

There are a variety of teaching approaches in standardized SBAR education. In this study, simulation 
learning was used because it is possible to present diverse examples of clinical cases and reproduce clinical situations that are similar to real life.

Education based on simulation can repeatedly provide an opportunity to train for risky situations in a condition without actual risk factors, accentuating the learning effect. Particularly, it reproduces rarely occurring clinical situations, making it possible for students to experience complex clinical situations[22].

In current practicum courses, it is very difficult for graduate nurses to directly experience complex emergency situations. It is believed that in SBAR education using simulation to reproduce a variety of emergency situations, students experience in-depth leaning through repeated learning in a safe environment to develop the competency to analyze patient situations accurately and communicate clearly and confidently based on the data, even in an emergency situation. Undergraduate nursing students should learn standardized communication skills while experiencing various and complex clinical situations through SBAR education programs using simulation. In this way, clinical nurses will be able to communicate clearly and confidently after graduation, to perform nursing work accurately, to collaborate with other medical staff, and to improve patient safety and nursing quality.

\section{Conclusion}

This single-group pre-post experimental study was conducted with 82 nursing students to examine the effects of the simulation-based SBAR education program on communication clarity and communication self-confidence. After undergraduate nursing students were provided with the SBAR education program based on simulation reproducing emergency situations, the clarity and selfconfidence in inter-medical staff communication were significantly improved. Both first and second hypotheses were supported by the improvement in communication clarity, and communication selfconfidence after learning than before the SBAR education program based on the simulation of emergency situations.

This study was conducted with a single group of participants taking a 15-week course in the regular curriculum. Accordingly, the findings should be interpreted with caution and repeated studies using the control group are required to consider the effects of learner maturity on the results of the study during the 15 weeks of the program. To date, the effects of SBAR education using simulation to reproduce emergency situations on long-term memory and in-depth learning have not been sufficiently investigated. Hence, future research should be conducted to examine such effects of simulation-based SBAR education.

Undergraduate nursing students improved their communication clarity and communication selfconfidence suitable for complex and emergency clinical situations through the SBAR education program using simulation. The course enabled clinical nurses to communicate clearly and confidently after graduation, collaborate with medical teams, and improve patient safety and nursing quality.

\section{Acknowledgments}

This research was supported by a 2020 Daegu University Research Grant.

\section{References}

[1] Y. B. Lee, M. S. Koh, The Effect of Clinical Nurses' Communication Competency and Emotional Intelligence on Organizational Performance, Journal of Korean Clinical Nursing Research, (2015), Vol.21, No.3, pp.347-354, DOI: http://dx.doi.org/10.22650/JKCNR.2015.21.3.347 
[2] Y. H. Kim, The job competence of new nurses expected in clinical, First Seminar on the Development of the National Examination Questions for Nurse, Korean Society of Nursing Science, pp.25-38, (2017), June 2, Seoul, South Korea.

[3] K. S. Kesten, Role-Play Using SBAR Technique to Improve Observed Communication Skills in Senior Nursing Students, Journal of Nursing Education, (2010), Vol.50, No.2, pp.79-87, DOI: http://dx.doi.org/10.3928/0148483420101230-02

[4] Y. S. Lee, Y. Eun, The Effect of the Flipped Learning on Self-efficacy, Critical Thinking Disposition, and Communication Competence of Nursing Students, Journal of Korean Academic Society of Nursing Education, (2016), Vol.22, No.4, pp.567-576, DOI: http://dx.doi.org/10.5977/jkasne.2016.22.4.567

[5] M. Randmaa, G. Martensson, C. L. Swenne, M. Engstrom, SBAR improves communication and safety climate and decreases incident reports due to communication errors in an anaesthetic clinic: a prospective intervention study, BMJ Open, (2014), Vol.4, No.1, e04268, DOI: http://dx.doi.org/10.1136/bmjopen-2013-004268

[6] M. Raymond, M. C. Harrison, The structured communication tool SBAR(Situation, Background, Assessment and Recommendation) improves communication in neonatology, South African Medical Journal, (2014), Vol.104, No.12, pp.850-852, DOI: $10.7196 /$ samj. 8684

[7] P. Cornell, M. T. Gervis, L. Yates, J. M. Vardaman, Improving Shift Report Focus and Consistency With the Situation, Background, Assessment, Recommendation Protocol, The Journal of Nursing Administration, (2013), Vol.43, No.7-8, pp.422-428, DOI: http://dx.doi.org/10.1097/NNA.0b013e31829d6303

[8] G. O. Noh, H. K. Son, D. H. Kim, Effect of SBAR Education Program Based on Simulation Practiceon Report Clarity and Confidence in Nursing Students, Korean J Health Commun., (2016), Vol.11, No.2, pp.145-153, DOI: http://dx.doi.org/10.15715/kjhcom.2016.11.2.145

[9] I. J. Seo, S. Y. Jang, Effects on Nursing Student's Communication Clarity, communication Confidence, and Self-efficacy Following SBAR Program, The Journal of Learner-Centered Curriculum and Instruction, (2020), Vol.20, No.1, pp.977992, DOI: http://dx.doi.org/10.22251/jlcci.2020.20.1.977

[10] J. H. Yoon, E. J. Lee, The Effect of Team Based Simulation Learning Using SBAR on Critical Thinking and Communication Clarity of Nursing Students, Journal of the Korean Academia-Industrial cooperation Society, (2018), Vol.19, No.9, pp.42-49, DOI: http://dx.doi.org/10.5762/KAIS.2018.19.9.42

[11] E. J. Kim, The effect of simulation-based SBAR training program on nursing students communication and clinical judgement, Cheonnam University, Ph.D. dissertation, pp.42-51, (2018)

[12] H. H. Cho, K. H. Nam, J. S. Park, H. E. Jeong, Y. J. Jung, The Effect of Simulation Training applying SBAR for Nursing Students on Communication Clarity, Self-Confidence in Communication, and Clinical Decision-Making Ability, Journal of the Korean Academia-Industrial Cooperation Society, (2020), Vol.21, No.7, pp.73-81, DOI: http://dx.doi.org/10.5762/KAIS.2020.21.7.73

[13] Y. H. Kim, Y. S. Choi, H. Y. Jun, M. J. Kim, Effects of SBAR Program on Communication Clarity, Clinical Competence and Self-efficacy for Nurses in Cancer Hospitals, The Korean Journal of Rehabilitation Nursing, (2016), Vol.19, No.1, pp.20-29, DOI: http://dx.doi.org/10.7587/kjrehn.2016.20

[14] J. Y. Lee, Effective communication for patient safety, Journal of the Korean Medical Association, (2015), Vol.58, No.2, pp.100-104, DOI: http://dx.doi.org/10.5124/jkma.2015.58.2.100

[15] J. Compton, K. Copeland, S. Flanders, C. Cassity, M. Spetman, Y. Xiao, D. Kennerly, Implementing SBAR Across a Large Multihospital Health System, The Joint Commission Journal of Quality and Patient Safety, (2012), Vol.38, No.6, pp.261-268, DOI: http://dx.doi.org/10.1016/S1553-7250(12)38033-1

[16] M. Yu, K. J. Kang, Effectiveness of a role-play simulation program involving the sbar technique: A quasi-experimental study, Nurse Education Today, (2017), Vol.53, pp.41-47, DOI: http://dx.doi.org/10.1016/j.nedt.2017.04.002

[17] H. J. Cho, The effect of SBAR program education on a nurse's communication clarify and self-expression, Yonsei University, Master's thesis, pp.25-58, (2013)

[18] W. J. Kim, S. Y. Kim, A Study on The Effects of The Nursing Management Practice using SBAR on Nursing Students' Self-expressiveness, Communication Skills, and Abilities to Think Critically, Journal of Learner-Centered Curriculum and Instruction, (2016), Vol.16, No.9, pp.457-472, DOI: http://dx.doi.org/10.22251/jlcci.2016.16.9.457 
[19] S. Marshall, J. Harrison, B. Flanagan, The teaching of a structured tool improves the clarity and content of interprofessional clinical communication, BMJ Quality \& Safety, (2009), Vol.18, No.2, pp.137-140, DOI: http://dx.doi.org/10.1136/qshc.2007.025247

[20] Y. H. Park, A study on the communication system model and recognition analysis of president-staffs communication in university, Wonkwang University, Ph.D. dissertation, pp.112-121(2010)

[21] J. K. Lee, The relationships among clinical competence, clinical practice stress, communication skill and nunchi in nursing students, Gyeongsang National University, Master's thesis, pp.30-35, (2015)

[22] W. Eppich, V. Howard, J. Vozenilek, I. Curran, Simulation-Based Team Training in Healthcare, Journal of the Society for Simulation in Healthcare, (2011), Vol.6, No.7, pp.S14-S19, DOI: http://dx.doi.org/10.1097/SIH.0b013e318229f550 